\title{
Existence Results for a Coupled System of Nonlinear Fractional Differential Equation with Four-Point Boundary Conditions
}

\author{
M. Gaber' and M. G. Brikaa ${ }^{2}$ \\ ${ }^{1}$ Faculty of Education Al-Arish, Suez Canal University, Ismailia 41522, Egypt \\ ${ }^{2}$ Faculty of Computers and Informatics, Suez Canal University, Ismailia 41522, Egypt \\ Correspondence should be addressed to M. G. Brikaa, gaber.brikaa@yahoo.com
}

Received 30 August 2011; Accepted 20 October 2011

Academic Editor: J.-L. Wu

Copyright (C) 2011 M. Gaber and M. G. Brikaa. This is an open access article distributed under the Creative Commons Attribution License, which permits unrestricted use, distribution, and reproduction in any medium, provided the original work is properly cited.

This paper studies a coupled system of nonlinear fractional differential equation with four-point boundary conditions. Applying the Schauder fixed-point theorem, an existence result is proved for the following system: $D^{\alpha} u(t)=f\left(t, v(t), D^{m} v(t)\right), t \in(0,1), D^{\beta} v(t)=f\left(t, u(t), D^{n} u(t)\right), t \in(0,1)$, $u(0)=\gamma u(\zeta), u(1)=\delta u(\eta), v(0)=\gamma v(\zeta), v(1)=\delta v(\eta)$, where $\alpha, \beta, m, n, \zeta, \eta, \gamma, \delta$ satisfy certain conditions.

\section{Introduction}

Fractional differential equations arise in many engineering and scientific disciplines as the mathematical modelling of systems and processes in the fields of physics, chemistry, aerodynamics, electrodynamics of complex medium, polymerrheology, and so forth involves derivatives of fractional order. Fractional differential equations also serve as an excellent tool for the description of hereditary properties of various materials and processes. In consequence, the subject of fractional differential equations is gaining much importance and attention. For details, see [1-8] and the refrences therein.

On the other hand, the study of coupled systems involving fractional differential equations is also important as such systems occur in various problems of applied nature, for instance, see $[9,10]$. Recently, in [11], the existence of nontrivial solutions was investigated for a coupled system of nonlinear fractional differential equations with two-point boundary conditions by using Schauder's fixed-point theorem. Reference [12] established the existence of a positive solution to a singular coupled system of fractional order. The existence of nontrivial solutions for a coupled system of nonlinear fractional differential equations with 
three-point boundary conditions was investigated in [13] by using Schauder's fixed point theorem.

In this paper, we consider a four-point boundary value problem for a coupled system of nonlinear fractional differential equation given by

$$
\begin{gathered}
D^{\alpha} u(t)=f\left(t, v(t), D^{m} v(t)\right), \quad t \in(0,1), \\
D^{\beta} v(t)=f\left(t, u(t), D^{n} u(t)\right), \quad t \in(0,1), \\
u(0)=\gamma u(\zeta), \quad u(1)=\delta u(\eta), \quad v(0)=\gamma v(\zeta), \quad v(1)=\delta v(\eta),
\end{gathered}
$$

where $1<\alpha, \beta<2, m, n, \gamma, \delta>0,0<\zeta<\eta<1, \alpha-n \geq 1, \beta-m \geq 1, \delta \eta^{\alpha-1}<1, \delta \eta^{\alpha-2}<1$, $\gamma \zeta^{\alpha-1}<1, \gamma \zeta^{\alpha-2}<1, \delta \eta^{\beta-1}<1, \delta \eta^{\beta-2}<1, \gamma \zeta^{\beta-1}<1, \gamma \zeta^{\beta-2}<1, D$ is the standard RiemannLiouville fractional derivative, and $f, g:[0,1] \times R \times R \rightarrow R$ are given continuous function.

The organization of this paper is as follows. In Section 2, we present some necessary definition and preliminary results that will be used to prove our main results. The proofs of our main results are given in Section 3.

\section{Preliminaries}

For the convenience of the reader, we present here the necessary definition from fractional calculus theory and preliminary results.

Definition 2.1 (see [5]). The Riemann-Liouville fractional integral of order $q>0$ of function $f:(0, \infty) \rightarrow R$ is given by

$$
I^{q} f(t)=\frac{1}{\lceil(q)} \int_{0}^{t} \frac{f(s)}{(t-s)^{1-q}} d s
$$

provided that the integral exists.

Definition 2.2 (see [5]). The Riemann-Liouville fractional derivative of order $q>0$ of function $f:(0, \infty) \rightarrow R$ is given by

$$
D^{q} f(t)=\frac{1}{\Gamma(n-q)}\left(\frac{d}{d t}\right)^{n} \int_{0}^{t} \frac{f(s)}{(t-s)^{q-n+1}} d s,
$$

where $n=[q]+1$ and $[q]$ denotes the integral part of number $q$, provided that the right side is pointwise defined on $(0, \infty)$.

Lemma 2.3 ([5]). Let $n-1<\alpha \leq n, D^{\alpha} u(t)$ exists for $t \in(0,1)$. Then,

$$
I^{\alpha} D^{\alpha} u(t)=u(t)+C_{1} t^{\alpha-1}+C_{2} t^{\alpha-2}+\cdots+C_{n} t^{\alpha-n} .
$$

Remark 2.4. The following properties are useful for our discussion: $I^{\alpha} I^{\beta} f(t)=I^{\alpha+\beta} f(t)$, $D^{\alpha} I^{\alpha} f(t)=f(t), \alpha>0, \beta>0, f \in L(0,1) ; I^{\alpha} D^{\alpha} f(t)=f(t), 0<\alpha<1, f(t) \in C[0,1]$ and $D^{\alpha} f(t) \in C(0,1) \cap L(0,1) ; I^{\alpha}: C[0,1] \rightarrow C[0,1], \alpha>0$. 
For convenience, we introduce the following notation. Let

$$
\begin{array}{llll}
\mu_{1}=\gamma \zeta^{\alpha-1}, & v_{1}=1-\gamma \zeta^{\alpha-2}, & \varpi_{1}=1-\delta \eta^{\alpha-1}, & \lambda_{1}=1-\delta \eta^{\alpha-2}, \\
\mu_{2}=\gamma \zeta^{\beta-1}, & v_{2}=1-\gamma \zeta^{\beta-2}, & \varpi_{2}=1-\delta \eta^{\beta-1}, & \lambda_{2}=1-\delta \eta^{\beta-2} .
\end{array}
$$

Let $C(J)$ Denote the space of all continuous functions defined on $J=[0,1]$. Let $X=\left\{u(t): u \in C(J)\right.$ and $\left.D^{n} u \in C(J)\right\}$ be a Banach space endowed with the norm $\|u\|_{x}=\max _{t \in J}|u(t)|+\max _{t \in J}\left|D^{n} u(t)\right|$, where $1<\alpha<2,0<n \leq \alpha-1$, see [11] Lemma 3.2, and let $Y=\left\{v(t): v \in C(J)\right.$ and $\left.D^{m} v \in C(J)\right\}$ be a Banach space equipped with the norm $\|v\|_{Y}=\max _{t \in J}|v(t)|+\max _{t \in J}\left|D^{m} v(t)\right|$, where $1<\beta<2,0<m \leq \beta-1$. Thus, $\left(X \times Y,\|\cdot\|_{X \times Y}\right)$ is a Banach with the norm defined by $\|(u, v)\|_{X \times Y}=\max \left\{\|u\|_{x},\|v\|_{Y}\right\}$ for $(u, v) \in X \times Y$.

Lemma 2.5. Let $y \in C(J)$ be a given function and $1<\alpha<2$. Then, the unique solution of

$$
\begin{gathered}
D^{\alpha} u(t)=y(t), \quad t \in(0,1), \\
u(0)=\gamma u(\zeta), \quad u(1)=\delta u(\eta),
\end{gathered}
$$

is given by

$$
u(t)=\int_{0}^{1} K_{1}(t, s) y(s) d s
$$

where $K_{1}(t, s)$ is Green's function given by

$$
\begin{gathered}
K_{1}(t, s)=\frac{1}{\Gamma(\alpha)\left(\varpi_{1} v_{1}+\mu_{1} \lambda_{1}\right)} \begin{cases}K_{11}(t, s), \quad 0 \leq t \leq \zeta, \\
K_{12}(t, s), \quad \zeta<t \leq \eta, \\
K_{13}(t, s), \quad \eta<t \leq 1,\end{cases} \\
K_{11}(t, s)= \begin{cases}{[t-s)^{\alpha-1}\left(\varpi_{1} v_{1}+\mu_{1} \lambda_{1}\right)+\gamma t^{\alpha-2}\left(\varpi_{1}-\lambda_{1} t\right)(\zeta-s)^{\alpha-1}} \\
\left.+\delta t^{\alpha-2}\left(v_{1} t+\mu_{1}\right)(\eta-s)^{\alpha-1}-t^{\alpha-2}\left(v_{1} t+\mu_{1}\right)(1-s)^{\alpha-1}\right], & 0 \leq s \leq t, \\
\left.-t^{\alpha-2}\left(v_{1} t+\mu_{1}\right)(1-s)^{\alpha-1}\right], & t<s \leq \zeta, \\
{\left[\delta t^{\alpha-2}\left(\varpi_{1} t+\mu_{1}\right)(\eta-s)^{\alpha-1}-t^{\alpha-2}\left(\nu_{1} t+\mu_{1}\right)(1-s)^{\alpha-1}\right]} & \zeta<s \leq \eta, \\
-t^{\alpha-2}\left(v_{1} t+\mu_{1}\right)(1-s)^{\alpha-1}, & \eta<s \leq 1,\end{cases}
\end{gathered}
$$




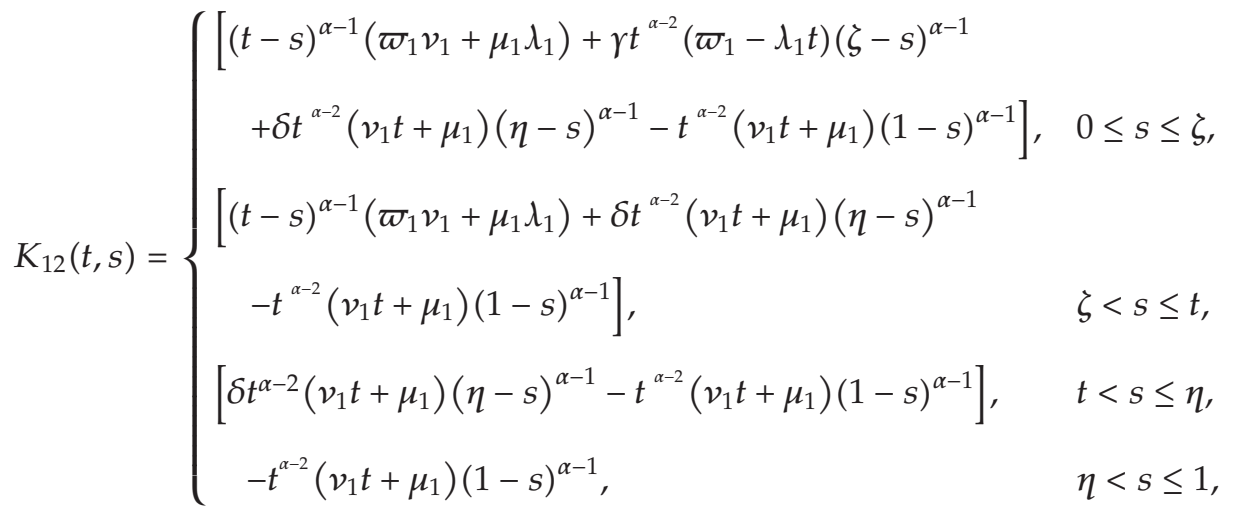

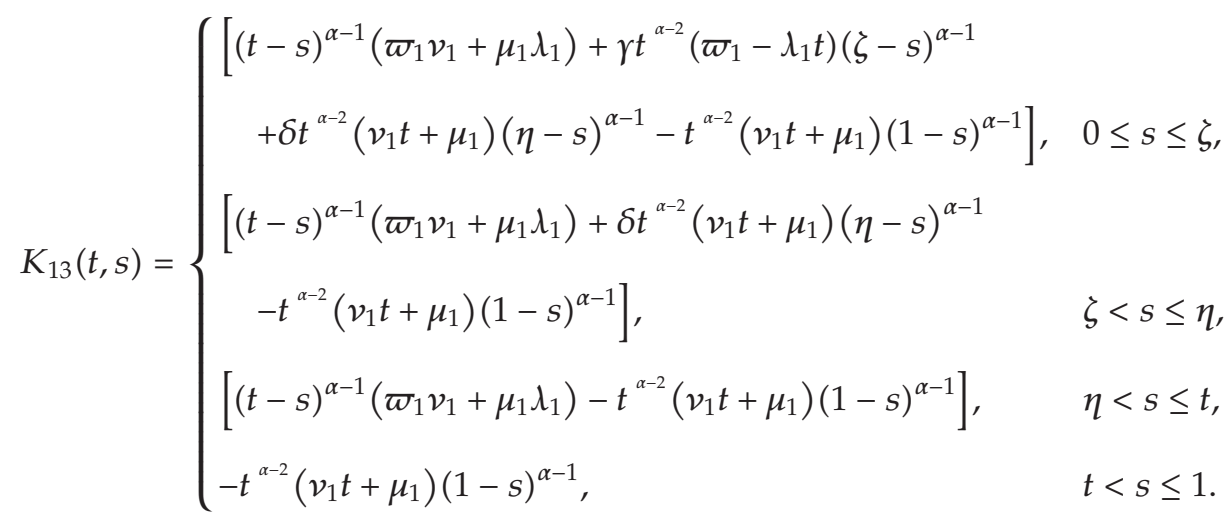

Proof. For $C_{1}, C_{2} \in R$, the general solution of (2.5) can be written as

$$
\begin{aligned}
u(t) & =I^{\alpha} y(t)+C_{1} t^{\alpha-1}+C_{2} t^{\alpha-2} \\
& =\int_{0}^{t} \frac{(t-s)^{\alpha-1}}{\Gamma(\alpha)} y(s) d s+C_{1} t^{\alpha-1}+C_{2} t^{\alpha-2} .
\end{aligned}
$$

By the boundary condition,

$$
\begin{gathered}
u(0)=r u(\zeta), \\
C_{2}=\frac{1}{v_{1}}\left(r \int_{0}^{\zeta} \frac{(\zeta-s)^{\alpha-1}}{\Gamma(\alpha)} y(s) d s+\mu_{1} C_{1}\right) .
\end{gathered}
$$

By the boundary condition,

$$
\begin{gathered}
u(1)=\delta u(\eta) \\
C_{1}=\frac{1}{\varpi_{1}}\left(\delta \int_{0}^{\eta} \frac{(\eta-s)^{\alpha-1}}{\Gamma(\alpha)} y(s) d s-\int_{0}^{1} \frac{(1-s)^{\alpha-1}}{\Gamma(\alpha)} y(s) d s-\lambda_{1} C_{2}\right) .
\end{gathered}
$$


Substituting (2.11) into (2.12), we get

$$
\begin{aligned}
C_{1}= & \frac{\delta v_{1}}{\left(\varpi_{1} v_{1}+\mu_{1} \lambda_{1}\right)} \int_{0}^{\eta} \frac{(\eta-s)^{\alpha-1}}{\Gamma(\alpha)} y(s) d s \\
& -\frac{v_{1}}{\left(\varpi_{1} v_{1}+\mu_{1} \lambda_{1}\right)} \int_{0}^{1} \frac{(1-s)^{\alpha-1}}{\Gamma(\alpha)} y(s) d s \\
& -\frac{\gamma \lambda_{1}}{\left(\varpi_{1} v_{1}+\mu_{1} \lambda_{1}\right)} \int_{0}^{\zeta} \frac{(\zeta-s)^{\alpha-1}}{\Gamma(\alpha)} y(s) d s
\end{aligned}
$$

Substituting (2.13) into (2.11), we get

$$
\begin{aligned}
C_{2}= & \frac{\gamma \varpi_{1}}{\left(\varpi_{1} v_{1}+\mu_{1} \lambda_{1}\right)} \int_{0}^{\zeta} \frac{(\zeta-s)^{\alpha-1}}{\Gamma(\alpha)} y(s) d s \\
& +\frac{\delta \mu_{1}}{\left(\varpi_{1} v_{1}+\mu_{1} \lambda_{1}\right)} \int_{0}^{\eta} \frac{(\eta-s)^{\alpha-1}}{\Gamma(\alpha)} y(s) d s \\
& -\frac{\mu_{1}}{\left(\varpi_{1} v_{1}+\mu_{1} \lambda_{1}\right)} \int_{0}^{1} \frac{(1-s)^{\alpha-1}}{\Gamma(\alpha)} y(s) d s .
\end{aligned}
$$

Thus, the unique solution of (2.5) and (2.6) is

$$
\begin{aligned}
u(t)= & \int_{0}^{t} \frac{(t-s)^{\alpha-1}}{\Gamma(\alpha)} y(s) d s \\
& +\frac{\gamma t^{\alpha-2}\left(\varpi_{1}-\lambda_{1} t\right)}{\left(\varpi_{1} v_{1}+\mu_{1} \lambda_{1}\right)} \int_{0}^{\zeta} \frac{(\zeta-s)^{\alpha-1}}{\Gamma(\alpha)} y(s) d s \\
& +\frac{\delta t^{\alpha-2}\left(v_{1} t+\mu_{1}\right)}{\left(\varpi_{1} v_{1}+\mu_{1} \lambda_{1}\right)} \int_{0}^{\eta} \frac{(\eta-s)^{\alpha-1}}{\Gamma(\alpha)} y(s) d s \\
& -\frac{t^{\alpha-2}\left(v_{1} t+\mu_{1}\right)}{\left(\varpi_{1} v_{1}+\mu_{1} \lambda_{1}\right)} \int_{0}^{1} \frac{(1-s)^{\alpha-1}}{\Gamma(\alpha)} y(s) d s \\
= & \int_{0}^{1} K_{1}(t, s) y(s) d s,
\end{aligned}
$$

where $K_{1}(t, s)$ is given by (2.8).

Similarly, the general solution of

$$
\begin{gathered}
D^{\beta} v(t)=y(t), \quad t \in(0,1), \\
v(0)=\gamma v(\zeta), \quad v(1)=\delta v(\eta),
\end{gathered}
$$


is

$$
v(t)=\int_{0}^{1} K_{2}(t, s) y(s) d s,
$$

where $K_{2}(t, s)$, can be obtained from $K_{1}(t, s)$ by replacing $\alpha$ with $\beta$. Let $\left(K_{1}, K_{2}\right)$ denote Green's function for the boundary value problem (1.1).

Consider the coupled system of integral equation:

$$
\begin{aligned}
& u(t)=\int_{0}^{1} K_{1}(t, s) f\left(s, v(s), D^{p} v(s)\right) d s, \\
& v(t)=\int_{0}^{1} K_{2}(t, s) g\left(s, u(s), D^{q} u(s)\right) d s .
\end{aligned}
$$

\section{Main Results}

Lemma 3.1. Assume that $f, g: J \times R \times R \rightarrow R$ are continuous functions. Then, $(u, v) \in X \times Y$ is a solution of (1.1) if and only if $(u, v) \in X \times Y$ is a solution of (2.18).

Proof. The proof is immediate from Lemma 2.5, so we omit it.

Let us define an operator $F: X \times Y \rightarrow X \times Y$ as

$$
F(u, v)(t)=\left(F_{1} v(t), F_{2} u(t)\right)
$$

where

$$
F_{1} v(t)=\int_{0}^{1} K_{1}(t, s) f\left(s, v(s), D^{p} v(s)\right) d s, \quad F_{2} u(t)=\int_{0}^{1} K_{2}(t, s) g\left(s, u(s), D^{q} u(s)\right) d s
$$

In view of the continuity of $K_{1}, K_{2}, f, g$, it follows that $F$ is continuous. Moreover, by Lemma 3.1, the fixed-point of the operator $F$ coincides with the solution of (1.1).

For the forthcoming analysis, we introduce the growth condition on $f$ and $g$ as

$\left(\mathrm{A}_{1}\right)$ there exists a nonnegative function $a(t) \in L(0,1)$ such that

$$
|f(t, x, y)| \leq a(t)+\epsilon_{1}|x|^{\rho_{1}}+\epsilon_{2}|y|^{\rho_{2}}, \quad \epsilon_{1}, \epsilon_{2}>0,0<\rho_{1}, \rho_{2}<1,
$$

$\left(\mathrm{A}_{2}\right)$ there exist a nonnegative function $b(t) \in L(0,1)$ such that

$$
|g(t, x, y)| \leq b(t)+\delta_{1}|x|^{\sigma_{1}}+\delta_{2}|y|^{\sigma_{2}}, \quad \delta_{1}, \delta_{2}>0,0<\sigma_{1}, \sigma_{2}<1 .
$$


Let us set the following notations for convenience:

$$
\begin{aligned}
& A=\frac{1}{\alpha \Gamma(\alpha+1)\left\lceil(\alpha-q+1)\left(\varpi_{1} v_{1}+\mu_{1} \lambda_{1}\right)\right.} \\
& \times\left[\gamma \zeta^{\alpha} \Gamma(\alpha+1)(\alpha-q)\left(\varpi_{1}-\lambda_{1}\right)+\delta \eta^{\alpha} \Gamma(\alpha+1)(\alpha-q)\left(v_{1}+\mu_{1}\right)\right. \\
& +\alpha \Gamma(\alpha+1)\left(\nu_{1}\left(1+\varpi_{1}\right)+\mu_{1}\left(1+\lambda_{1}\right)\right) \\
& +\alpha\left[(\alpha-q+1)\left(\left(1-\delta \eta^{\alpha}\right)\left(\nu_{1}+\mu_{1}\right)-\gamma \zeta^{\alpha}\left(\varpi_{1}-\lambda_{1}\right)\right)\right], \\
& B=\frac{1}{\beta \Gamma(\beta+1) \Gamma(\beta-p+1)\left(\varpi_{2} v_{2}+\mu_{2} \lambda_{2}\right)} \\
& \times\left[\gamma \zeta^{\beta} \Gamma(\beta+1)(\beta-p)\left(\varpi_{2}-\lambda_{2}\right)+\delta \eta^{\beta} \Gamma(\beta+1)(\beta-p)\left(v_{2}+\mu_{2}\right)\right. \\
& +\beta \Gamma(\beta+1)\left(v_{2}\left(1+\varpi_{2}\right)+\mu_{2}\left(1+\lambda_{2}\right)\right) \\
& +\beta\left[(\beta-p+1)\left(\left(1-\delta \eta^{\beta}\right)\left(\nu_{2}+\mu_{2}\right)-\gamma \zeta^{\beta}\left(\varpi_{2}-\lambda_{2}\right)\right)\right], \\
& m=\max _{t \in J} \int_{0}^{1}\left|a(s) k_{1}(t, s)\right| d s+\frac{1}{\left\lceil(\alpha-q)\left(\varpi_{1} \nu_{1}+\mu_{1} \lambda_{1}\right)\right.} \\
& \times\left[\gamma\left(\varpi_{1}-\lambda_{1}\right) \int_{0}^{\zeta}(\zeta-s)^{\alpha-1} a(s) d s+\delta\left(\nu_{1}+\mu_{1}\right) \int_{0}^{\eta}(\eta-s)^{\alpha-1} a(s) d s\right. \\
& \left.+\left(v_{1}\left(1+\varpi_{1}\right)+\mu_{1}\left(1+\lambda_{1}\right)\right) \int_{0}^{1}(1-s)^{\alpha-q-1} a(s) d s\right], \\
& n=\max _{t \in J} \int_{0}^{1}\left|b(s) k_{1}(t, s)\right| d s+\frac{1}{\left\lceil(\beta-p)\left(\varpi_{2} v_{2}+\mu_{2} \lambda_{2}\right)\right.} \\
& \times\left[\gamma\left(\varpi_{2}-\lambda_{2}\right) \int_{0}^{\zeta}(\zeta-s)^{\beta-1} b(s) d s+\delta\left(\nu_{2}+\mu_{2}\right) \int_{0}^{\eta}(\eta-s)^{\beta-1} b(s) d s\right. \\
& \left.+\left(v_{2}\left(1+\varpi_{2}\right)+\mu_{2}\left(1+\lambda_{2}\right)\right) \int_{0}^{1}(1-s)^{\beta-p-1} b(s) d s\right] .
\end{aligned}
$$

Define a ball $W$ in the Banach space $X \times Y$ as

$$
W=\left\{(u(t), v(t)) \mid(u(t), v(t)) \in X \times Y,\|(u(t), v(t))\|_{X \times Y} \leq R, t \in J\right\},
$$

where $R \geq \max \left\{\left(3 A \epsilon_{1}\right)^{1 /\left(1-\rho_{1}\right)},\left(3 A \epsilon_{2}\right)^{1 /\left(1-\rho_{2}\right)},\left(3 B \delta_{1}\right)^{1 /\left(1-\sigma_{1}\right)},\left(3 B \delta_{2}\right)^{1 /\left(1-\sigma_{2}\right)}, 3 m, 3 n\right\}$.

Theorem 3.2. Assume that the assumptions $\left(\mathrm{A}_{1}\right)$ and $\left(\mathrm{A}_{2}\right)$ hold. Then, there exists a solution for the four-point boundary value problem (1.1). 
Proof. As a first step, we prove that $F: W \rightarrow W$ :

$$
\begin{aligned}
& \left|F_{1} v(t)\right|=\left|\int_{0}^{1} k_{1}(t, s) f\left(s, v(s), D^{p} v(s)\right) d s\right| \\
& \leq \int_{0}^{1}\left|a(s) k_{1}(t, s)\right| d s+\left(\epsilon_{1}|R|^{\rho_{1}}+\epsilon_{2}|R|^{\rho_{2}}\right) \int_{0}^{1}\left|k_{1}(t, s)\right| d s \\
& =\int_{0}^{1}\left|a(s) k_{1}(t, s)\right| d s+\left(\epsilon_{1}|R|^{\rho_{1}}+\epsilon_{2}|R|^{\rho_{2}}\right) \\
& \times\left(-\int_{0}^{t} \frac{(t-s)^{\alpha-1}}{\Gamma(\alpha)} d s-\frac{\gamma t^{\alpha-2}\left(\varpi_{1}-\lambda_{1} t\right)}{\left(\varpi_{1} v_{1}+\mu_{1} \lambda_{1}\right)} \int_{0}^{\zeta} \frac{(\zeta-s)^{\alpha-1}}{\Gamma(\alpha)} d s\right. \\
& \left.-\frac{\delta t^{\alpha-2}\left(\nu_{1} t+\mu_{1}\right)}{\left(\varpi_{1} v_{1}+\mu_{1} \lambda_{1}\right)} \int_{0}^{\eta} \frac{(\eta-s)^{\alpha-1}}{\Gamma(\alpha)} d s+\frac{t^{\alpha-2}\left(\nu_{1} t+\mu_{1}\right)}{\left(\varpi_{1} v_{1}+\mu_{1} \lambda_{1}\right)} \int_{0}^{1} \frac{(1-s)^{\alpha-1}}{\Gamma(\alpha)} d s\right) \\
& =\int_{0}^{1}\left|a(s) k_{1}(t, s)\right| d s+\left(\epsilon_{1}|R|^{\rho_{1}}+\epsilon_{2}|R|^{\rho_{2}}\right) \\
& \times\left(-\frac{t^{\alpha}}{\Gamma(\alpha+1)}+\frac{t^{\alpha-2}\left(v_{1} t+\mu_{1}\right)-\gamma \zeta^{\alpha} t^{\alpha-2}\left(\varpi_{1}-\lambda_{1} t\right)-\delta \eta^{\alpha} t^{\alpha-2}\left(\nu_{1} t+\mu_{1}\right)}{\left(\varpi_{1} v_{1}+\mu_{1} \lambda_{1}\right) \Gamma(\alpha+1)}\right) \\
& \leq \int_{0}^{1}\left|a(s) k_{1}(t, s)\right| d s+\left(\epsilon_{1}|R|^{\rho_{1}}+\epsilon_{2}|R|^{\rho_{2}}\right) \\
& \times\left(\frac{\left(1-\delta \eta^{\alpha}\right)\left(\nu_{1}+\mu_{1}\right)-\gamma \zeta^{\alpha}\left(\varpi_{1}-\lambda_{1}\right)}{\left(\varpi_{1} v_{1}+\mu_{1} \lambda_{1}\right) \Gamma(\alpha+1)}\right) \\
& \left|D^{q} F_{1} v(t)\right|=\mid D^{q} I^{\alpha} f\left(t, v(t), D^{p} v(t)\right)+\frac{1}{\left(\varpi_{1} v_{1}+\mu_{1} \lambda_{1}\right)} \\
& \times\left[r\left(\varpi_{1} D^{q} t^{\alpha-2}-\lambda_{1} D^{q} t^{\alpha-1}\right) I^{\alpha} f\left(\zeta, v(\zeta), D^{p} v(\zeta)\right)\right. \\
& +\delta\left(v_{1} D^{q} t^{\alpha-1}+\mu_{1} D^{q} t^{\alpha-2}\right) I^{\alpha} f\left(\eta, v(\eta), D^{p} v(\eta)\right) \\
& \left.-\left(v_{1} D^{q} t^{\alpha-1}+\mu_{1} D^{q} t^{\alpha-2}\right) I^{\alpha} f\left(1, v(1), D^{p} v(1)\right)\right] \\
& \leq \int_{0}^{t} \frac{(t-s)^{\alpha-q-1}}{\Gamma(\alpha-q)} f\left(s, v(s), D^{p} v(s)\right) d s+\frac{1}{\left\lceil(\alpha-q)\left(\varpi_{1} v_{1}+\mu_{1} \lambda_{1}\right)\right.} \\
& \times\left[r\left(\varpi_{1}-\lambda_{1}\right) \int_{0}^{\zeta}(\zeta-s)^{\alpha-1} f\left(s, v(s), D^{p} v(s)\right) d s\right. \\
& +\delta\left(v_{1}+\mu_{1}\right) \int_{0}^{\eta}(\eta-s)^{\alpha-1} f\left(s, v(s), D^{p} v(s)\right) d s \\
& \left.+\left(v_{1}+\mu_{1}\right) \int_{0}^{1}(1-s)^{\alpha-1} f\left(s, v(s), D^{p} v(s)\right) d s\right]
\end{aligned}
$$




$$
\begin{aligned}
& \leq \frac{1}{\Gamma(\alpha-q)}\left[\int_{0}^{t}(t-s)^{\alpha-q-1} a(s) d s+\left(\epsilon_{1}|R|^{\rho_{1}}+\epsilon_{2}|R|^{\rho_{2}}\right) \int_{0}^{t}(t-s)^{\alpha-q-1} d s\right] \\
& +\frac{1}{\left\lceil(\alpha-q)\left(\varpi_{1} v_{1}+\mu_{1} \lambda_{1}\right)\right.} \\
& \times\left[\gamma\left(\varpi_{1}-\lambda_{1}\right) \int_{0}^{\zeta}(\zeta-s)^{\alpha-1} a(s) d s+\gamma\left(\varpi_{1}-\lambda_{1}\right)\left(\epsilon_{1}|R|^{\rho_{1}}+\epsilon_{2}|R|^{\rho_{2}}\right)\right. \\
& \times \int_{0}^{\zeta}(\zeta-s)^{\alpha-1} d s \\
& +\delta\left(v_{1}+\mu_{1}\right) \int_{0}^{\eta}(\eta-s)^{\alpha-1} a(s) d s+\delta\left(v_{1}+\mu_{1}\right)\left(\epsilon_{1}|R|^{\rho_{1}}+\epsilon_{2}|R|^{\rho_{2}}\right) \\
& \times \int_{0}^{\eta}(\eta-s)^{\alpha-1} d s \\
& +\left(v_{1}+\mu_{1}\right) \int_{0}^{1}(1-s)^{\alpha-1} a(s) d s+\left(v_{1}+\mu_{1}\right)\left(\epsilon_{1}|R|^{\rho_{1}}+\epsilon_{2}|R|^{\rho_{2}}\right) \\
& \left.\times \int_{0}^{1}(1-s)^{\alpha-1} d s\right] \\
& \leq \frac{1}{\left\lceil(\alpha-q)\left(\varpi_{1} v_{1}+\mu_{1} \lambda_{1}\right)\right.} \\
& \times\left[\gamma\left(\varpi_{1}-\lambda_{1}\right) \int_{0}^{\zeta}(\zeta-s)^{\alpha-1} a(s) d s+\gamma\left(\varpi_{1}-\lambda_{1}\right)\left(\epsilon_{1}|R|^{\rho_{1}}+\epsilon_{2}|R|^{\rho_{2}}\right)\right. \\
& \times \int_{0}^{\zeta}(\zeta-s)^{\alpha-1} d s \\
& +\delta\left(v_{1}+\mu_{1}\right) \int_{0}^{\eta}(\eta-s)^{\alpha-1} a(s) d s+\delta\left(v_{1}+\mu_{1}\right)\left(\epsilon_{1}|R|^{\rho_{1}}+\epsilon_{2}|R|^{\rho_{2}}\right) \\
& \times \int_{0}^{\eta}(\eta-s)^{\alpha-1} d s \\
& +\left(v_{1}\left(1+\varpi_{1}\right)+\mu_{1}\left(1+\lambda_{1}\right)\right)\left(\int_{0}^{1}(1-s)^{\alpha-1} a(s) d s+\left(\epsilon_{1}|R|^{\rho_{1}}+\epsilon_{2}|R|^{\rho_{2}}\right)\right. \\
& \left.\left.\times \int_{0}^{1}(1-s)^{\alpha-1} d s\right)\right] \\
& =\frac{1}{\left\lceil(\alpha-q)\left(\varpi_{1} v_{1}+\mu_{1} \lambda_{1}\right)\right.} \\
& \times\left[\gamma\left(\varpi_{1}-\lambda_{1}\right) \int_{0}^{\zeta}(\zeta-s)^{\alpha-1} a(s) d s+\delta\left(v_{1}+\mu_{1}\right) \int_{0}^{\eta}(\eta-s)^{\alpha-1} a(s) d s\right. \\
& \left.+\left(v_{1}\left(1+\varpi_{1}\right)+\mu_{1}\left(1+\lambda_{1}\right)\right) \int_{0}^{1}(1-s)^{\alpha-q-1} a(s) d s\right]
\end{aligned}
$$




$$
\begin{aligned}
& +\left(\epsilon_{1}|R|^{\rho_{1}}+\epsilon_{2}|R|^{\rho_{2}}\right) \\
& \times\left[\frac{\gamma \zeta^{\alpha}(\alpha-q)\left(\varpi_{1}-\lambda_{1}\right)+\delta \eta^{\alpha}(\alpha-q)\left(v_{1}+\mu_{1}\right)+\alpha\left(v_{1}\left(1+\varpi_{1}\right)+\mu_{1}\left(1+\lambda_{1}\right)\right)}{\alpha\left\lceil(\alpha-q+1)\left(\varpi_{1} v_{1}+\mu_{1} \lambda_{1}\right)\right.}\right] .
\end{aligned}
$$

Thus,

$$
\begin{aligned}
\left\|F_{1} v(t)\right\|_{x} & =\max _{t \in J}\left|F_{1} v(t)\right|+\max _{t \in J}\left|D^{q} F_{1} v(t)\right| \leq m+\left(\epsilon_{1}|R|^{\rho_{1}}+\epsilon_{2}|R|^{\rho_{2}}\right) A \\
& \leq \frac{R}{3}+\frac{R}{3}+\frac{R}{3}=R .
\end{aligned}
$$

Similarly, it can be shown that $\left\|F_{2} v(t)\right\|_{y} \leq n+\left(\epsilon_{1}|R|^{\rho_{1}}+\epsilon_{2}|R|^{\rho_{2}}\right) B \leq R$. Hence, we conclude that $\left\|F_{1} v(t)\right\|_{x \times y} \leq R$.

Since $F_{1} v(t), F_{2} v(t), D^{q} F_{1} v(t), D^{q} F_{2} v(t)$ are continuous on $J$, therefore, $F: W \rightarrow W$.

Now, we show that $F$ is a completely continuous operator. For that we fix

$$
M=\max _{t \in J}\left|f\left(t, v(t), D^{p} v(t)\right)\right|, \quad N=\max _{t \in J}\left|g\left(t, u(t), D^{p} v(t)\right)\right| .
$$

For $(u, v) \in W, t, \tau \in J(t<\tau)$, we have

$$
\begin{aligned}
& \left|F_{1} v(t)-F_{1} v(\tau)\right| \\
& =\left|\int_{0}^{1}\left(k_{1}(t, s)-k_{1}(\tau, s)\right) f\left(s, v(s), D^{p} v(s)\right) d s\right| \\
& \leq M\left[\int_{0}^{t}\left|\left(k_{1}(t, s)-k_{1}(\tau, s)\right)\right| d s+\int_{t}^{\tau}\left|\left(k_{1}(t, s)-k_{1}(\tau, s)\right)\right| d s+\int_{\tau}^{\zeta}\left|\left(k_{1}(t, s)-k_{1}(\tau, s)\right)\right| d s\right. \\
& \left.\quad+\int_{\zeta}^{\eta}\left|\left(k_{1}(t, s)-k_{1}(\tau, s)\right)\right| d s+\int_{\eta}^{1}\left|\left(k_{1}(t, s)-k_{1}(\tau, s)\right)\right| d s\right] \\
& =\frac{M}{\Gamma(\alpha)\left(\varpi_{1} v_{1}+\mu_{1} \lambda_{1}\right)} \\
& \quad \times\left[\int _ { 0 } ^ { t } \left\{\left((\tau-s)^{\alpha-1}-(t-s)^{\alpha-1}\right)\left[\varpi_{1} v_{1}+\mu_{1} \lambda_{1}\right]\right.\right. \\
& \quad+\left(\tau^{\alpha-1}-t^{\alpha-1}\right)\left[v_{1}(1-s)^{\alpha-1}+\gamma \lambda_{1}(\zeta-s)^{\alpha-1}-\delta v_{1}(\eta-s)^{\alpha-1}\right] \\
& \left.\quad+\left(\tau^{\alpha-2}-t^{\alpha-2}\right)\left[\mu_{1}(1-s)^{\alpha-1}-\gamma \varpi_{1}(\zeta-s)^{\alpha-1}-\delta \mu_{1}(\eta-s)^{\alpha-1}\right]\right\} d s
\end{aligned}
$$


ISRN Mathematical Analysis

11

$$
\begin{aligned}
& +\int_{t}^{\tau}\left\{(\tau-s)^{\alpha-1}\left[\varpi_{1} v_{1}+\mu_{1} \lambda_{1}\right]+\left(\tau^{\alpha-1}-t^{\alpha-1}\right)\left[v_{1}(1-s)^{\alpha-1}+\gamma \lambda_{1}(\zeta-s)^{\alpha-1}-\delta v_{1}(\eta-s)^{\alpha-1}\right]\right. \\
& \left.+\left(\tau^{\alpha-2}-t^{\alpha-2}\right)\left[\mu_{1}(1-s)^{\alpha-1}-\gamma \varpi_{1}(\zeta-s)^{\alpha-1}-\delta \mu_{1}(\eta-s)^{\alpha-1}\right]\right\} d s \\
& +\int_{\tau}^{\zeta}\left\{\left(\tau^{\alpha-1}-t^{\alpha-1}\right)\left[v_{1}(1-s)^{\alpha-1}+\gamma \lambda_{1}(\zeta-s)^{\alpha-1}-\delta v_{1}(\eta-s)^{\alpha-1}\right]\right. \\
& \left.+\left(\tau^{\alpha-2}-t^{\alpha-2}\right)\left[\mu_{1}(1-s)^{\alpha-1}-\gamma \varpi_{1}(\zeta-s)^{\alpha-1}-\delta \mu_{1}(\eta-s)^{\alpha-1}\right]\right\} d s \\
& +\int_{\zeta}^{\eta}\left\{\left(\tau^{\alpha-1}-t^{\alpha-1}\right)\left[v_{1}(1-s)^{\alpha-1}-\delta v_{1}(\eta-s)^{\alpha-1}\right]\right. \\
& \left.+\left(\tau^{\alpha-2}-t^{\alpha-2}\right)\left[\mu_{1}(1-s)^{\alpha-1}-\delta \mu_{1}(\eta-s)^{\alpha-1}\right]\right\} d s \\
& \left.+\int_{\eta}^{1}\left\{\left(\tau^{\alpha-1}-t^{\alpha-1}\right)\left[v_{1}(1-s)^{\alpha-1}\right]+\left(\tau^{\alpha-2}-t^{\alpha-2}\right)\left[\mu_{1}(1-s)^{\alpha-1}\right]\right\} d s\right] \\
& =\frac{M}{\Gamma(\alpha)\left(\varpi_{1} v_{1}+\mu_{1} \lambda_{1}\right)} \\
& \times\left[\int_{0}^{\tau}(\tau-s)^{\alpha-1}\left[\varpi_{1} v_{1}+\mu_{1} \lambda_{1}\right] d s-\int_{0}^{t}(t-s)^{\alpha-1}\left[\varpi_{1} v_{1}+\mu_{1} \lambda_{1}\right] d s\right. \\
& +\int_{0}^{\zeta}\left[\left(\tau^{\alpha-1}-t^{\alpha-1}\right)\left[\gamma \lambda_{1}(\zeta-s)^{\alpha-1}\right]-\left(\tau^{\alpha-2}-t^{\alpha-2}\right)\left[\gamma \varpi_{1}(\zeta-s)^{\alpha-1}\right]\right] d s \\
& +\int_{0}^{\eta}\left[\left(\tau^{\alpha-1}-t^{\alpha-1}\right)\left[v_{1}(1-s)^{\alpha-1}-\delta v_{1}(\eta-s)^{\alpha-1}\right]\right. \\
& \left.+\left(\tau^{\alpha-2}-t^{\alpha-2}\right)\left[\mu_{1}(1-s)^{\alpha-1}-\delta \mu_{1}(\eta-s)^{\alpha-1}\right]\right] d s \\
& \left.+\int_{\eta}^{1}\left[\left(\tau^{\alpha-1}-t^{\alpha-1}\right)\left[v_{1}(1-s)^{\alpha-1}\right]+\left(\tau^{\alpha-2}-t^{\alpha-2}\right)\left[\mu_{1}(1-s)^{\alpha-1}\right]\right] d s\right] \\
& =\frac{M}{\Gamma(\alpha+1)\left(\varpi_{1} v_{1}+\mu_{1} \lambda_{1}\right)} \\
& \times\left[\left(\tau^{\alpha}-t^{\alpha}\right)\left[\varpi_{1} v_{1}+\mu_{1} \lambda_{1}\right]+\left(\tau^{\alpha-1}-t^{\alpha-1}\right)\left[\gamma \zeta^{\alpha}\left(\lambda_{1}-\varpi_{1}\right)+\left(v_{1}+\mu_{1}\right)\left(1-\delta \eta^{\alpha}\right)\right]\right], \\
& \left|D^{q} F_{1} v(t)-D^{q} F_{1} v(\tau)\right| \\
& =\mid D^{q} I^{\alpha} f\left(t, v(t), D^{p} v(t)\right)+\frac{1}{\left(\varpi_{1} v_{1}+\mu_{1} \lambda_{1}\right)} \\
& \times\left[\gamma\left(\varpi_{1} D^{q} t^{\alpha-2}-\lambda_{1} D^{q} t^{\alpha-1}\right) I^{\alpha} f\left(\zeta, v(\zeta), D^{p} v(\zeta)\right)\right. \\
& +\delta\left(v_{1} D^{q} t^{\alpha-1}+\mu_{1} D^{q} t^{\alpha-2}\right) I^{\alpha} f\left(\eta, v(\eta), D^{p} v(\eta)\right)
\end{aligned}
$$


12

ISRN Mathematical Analysis

$$
\begin{aligned}
& \left.-\left(v_{1} D^{q} t^{\alpha-1}+\mu_{1} D^{q} t^{\alpha-2}\right) I^{\alpha} f\left(1, v(1), D^{p} v(1)\right)\right] \\
& -D^{q} I^{\alpha} f\left(\tau, v(\tau), D^{p} v(\tau)\right)-\frac{1}{\left(\varpi_{1} v_{1}+\mu_{1} \lambda_{1}\right)} \\
& \times\left[r\left(\varpi_{1} D^{q} \tau^{\alpha-2}-\lambda_{1} D^{q} \tau^{\alpha-1}\right) I^{\alpha} f\left(\zeta, v(\zeta), D^{p} v(\zeta)\right)\right. \\
& +\delta\left(v_{1} D^{q} \tau^{\alpha-1}+\mu_{1} D^{q} \tau^{\alpha-2}\right) I^{\alpha} f\left(\eta, v(\eta), D^{p} v(\eta)\right) \\
& \left.-\left(v_{1} D^{q} \tau^{\alpha-1}+\mu_{1} D^{q} \tau^{\alpha-2}\right) I^{\alpha} f\left(1, v(1), D^{p} v(1)\right)\right] \\
& \leq \frac{1}{\Gamma(\alpha-q)}\left[\left|\int_{0}^{t}(t-s)^{\alpha-q-1} f\left(s, v(s), D^{p} v(s)\right) d s-\int_{0}^{\tau}(\tau-s)^{\alpha-q-1} f\left(s, v(s), D^{p} v(s)\right) d s\right|\right] \\
& +\frac{M}{\left\lceil(\alpha-q)\left(\varpi_{1} v_{1}+\mu_{1} \lambda_{1}\right)\right.} \\
& \times\left|\left(t^{\alpha-q-1}-\tau^{\alpha-q-1}\right)\left\{-\gamma \lambda_{1} \int_{0}^{\zeta}(\zeta-s)^{\alpha-1} d s+\delta v_{1} \int_{0}^{\eta}(\eta-s)^{\alpha-1} d s-v_{1} \int_{0}^{1}(1-s)^{\alpha-1} d s\right\}\right| \\
& +\frac{M}{\alpha\left\lceil(\alpha-q-1)\left(\varpi_{1} v_{1}+\mu_{1} \lambda_{1}\right)\right.} \\
& \times\left|\left(t^{\alpha-q-2}-\tau^{\alpha-q-2}\right)\left\{r \varpi_{1} \int_{0}^{\zeta}(\zeta-s)^{\alpha-1} d s+\delta \mu_{1} \int_{0}^{\eta}(\eta-s)^{\alpha-1} d s-\mu_{1} \int_{0}^{1}(1-s)^{\alpha-1} d s\right\}\right| \\
& \leq \frac{1}{\Gamma(\alpha-q)}\left[\left|\int_{0}^{t}(t-s)^{\alpha-q-1} f\left(s, v(s), D^{p} v(s)\right) d s-\int_{0}^{\tau}(t-s)^{\alpha-q-1} f\left(s, v(s), D^{p} v(s)\right) d s\right|\right. \\
& \left.+\left|\int_{0}^{\tau}(t-s)^{\alpha-q-1} f\left(s, v(s), D^{p} v(s)\right) d s-\int_{0}^{\tau}(\tau-s)^{\alpha-q-1} f\left(s, v(s), D^{p} v(s)\right) d s\right|\right] \\
& +\frac{M\left(\gamma \zeta^{\alpha} \lambda_{1}+v_{1}\left(1-\delta \eta^{\alpha}\right)\right)}{\alpha\left\lceil(\alpha-q)\left(\varpi_{1} v_{1}+\mu_{1} \lambda_{1}\right)\right.}\left(t^{\alpha-q-1}-\tau^{\alpha-q-1}\right) \\
& +\frac{M\left(\gamma \zeta^{\alpha} \varpi_{1}+\mu_{1}\left(1-\delta \eta^{\alpha}\right)\right)}{\alpha^{2}\left\lceil(\alpha-q-1)\left(\varpi_{1} v_{1}+\mu_{1} \lambda_{1}\right)\right.}\left(t^{\alpha-q-2}-\tau^{\alpha-q-2}\right) \\
& \leq \frac{M}{\Gamma(\alpha-q)}\left[\int_{0}^{\tau}\left((\tau-s)^{\alpha-q-1}-(t-s)^{\alpha-q-1}\right) d s+\int_{t}^{\tau}(t-s)^{\alpha-q-1} d s\right] \\
& +\frac{M\left(\gamma \zeta^{\alpha} \lambda_{1}+\nu_{1}\left(1-\delta \eta^{\alpha}\right)\right)}{\alpha \Gamma(\alpha-q)\left(\varpi_{1} v_{1}+\mu_{1} \lambda_{1}\right)}\left(t^{\alpha-q-1}-\tau^{\alpha-q-1}\right) \\
& +\frac{M\left(\gamma \zeta^{\alpha} \varpi_{1}+\mu_{1}\left(1-\delta \eta^{\alpha}\right)\right)}{\alpha^{2}\left\lceil(\alpha-q-1)\left(\varpi_{1} v_{1}+\mu_{1} \lambda_{1}\right)\right.}\left(t^{\alpha-q-2}-\tau^{\alpha-q-2}\right) \\
& =\frac{M}{\Gamma(\alpha-q+1)}\left(\tau^{\alpha-q}-t^{\alpha-q}\right)
\end{aligned}
$$




$$
\begin{aligned}
& +\frac{M\left(\gamma \zeta^{\alpha} \lambda_{1}+\nu_{1}\left(1-\delta \eta^{\alpha}\right)\right)}{\alpha\left\lceil(\alpha-q)\left(\varpi_{1} \nu_{1}+\mu_{1} \lambda_{1}\right)\right.}\left(t^{\alpha-q-1}-\tau^{\alpha-q-1}\right) \\
& +\frac{M\left(\gamma \zeta^{\alpha} \varpi_{1}+\mu_{1}\left(1-\delta \eta^{\alpha}\right)\right)}{\alpha^{2}\left\lceil(\alpha-q-1)\left(\varpi_{1} \nu_{1}+\mu_{1} \lambda_{1}\right)\right.}\left(t^{\alpha-q-2}-\tau^{\alpha-q-2}\right) .
\end{aligned}
$$

Analogously, it can be proved that

$$
\begin{aligned}
\mid F_{1} u(t) & -F_{1} u(\tau) \mid \\
\leq & \frac{N}{\Gamma(\beta+1)\left(\varpi_{2} v_{2}+\mu_{2} \lambda_{2}\right)} \\
& \times\left[\left(\tau^{\beta}-t^{\beta}\right)\left[\varpi_{2} v_{2}+\mu_{2} \lambda_{2}\right]+\left(\tau^{\beta-1}-t^{\beta-1}\right)\left[\gamma \zeta^{\beta}\left(\lambda_{2}-\varpi_{2}\right)+\left(v_{2}+\mu_{2}\right)\left(1-\delta \eta^{\beta}\right)\right]\right] \\
\left|D^{p} F_{1} u(t)-D^{p} F_{1} u(\tau)\right| & \frac{N}{\Gamma(\beta-p+1)}\left(\tau^{\beta-p}-t^{\beta-p}\right) \\
& +\frac{N\left(\gamma \zeta^{\beta} \lambda_{2}+v_{2}\left(1-\delta \eta^{\beta}\right)\right)}{\beta \Gamma(\beta-p)\left(\varpi_{2} v_{2}+\mu_{2} \lambda_{2}\right)}\left(t^{\beta-p-1}-\tau^{\beta-p-1}\right) \\
& +\frac{N\left(\gamma \zeta^{\beta} \varpi_{2}+\mu_{2}\left(1-\delta \eta^{\beta}\right)\right)}{\beta^{2} \Gamma(\beta-p-1)\left(\varpi_{2} v_{2}+\mu_{2} \lambda_{2}\right)}\left(t^{\beta-p-2}-\tau^{\beta-p-2}\right) .
\end{aligned}
$$

Since the functions $t^{\alpha}, t^{\alpha-1}, t^{\beta}, t^{\beta-1}, t^{\alpha-q}, t^{\alpha-q-1}, t^{\beta-p}, t^{\beta-p-1}$ are uniformly continuous on $J$, therefore, it follows from the above estimates that $F W$ is an equicontinuous set. Also, it is uniformly bounded as $F W \subset W$. Thus, we conclude that $F$ is a completely continuous operator. Hence, by Schauder's fixed-point theorem, there exists a solution for the four-point boundary value problem (1.1).

\section{References}

[1] A. Babakhani and V. D. Gejji, "Existence of positive solutions of nonlinear fractional differential equations," Journal of Mathematical Analysis and Applications, vol. 278, no. 2, pp. 434-442, 2003.

[2] Z. Bai and H. Lu, "Positive solutions for boundary value problem of nonlinear fractional differential equation," Journal of Mathematical Analysis and Applications, vol. 311, no. 2, pp. 495-505, 2005.

[3] M. El-Shahed and J. J. Nieto, "Nontrivial solutions for a nonlinear multi-point boundary value problem of fractional order," Computers E Mathematics with Applications, vol. 59, no. 11, pp. 3438-3443, 2010.

[4] T. Jankowski, "Positive solutions to second order four-point boundary value problems for impulsive differential equations," Applied Mathematics and Computation, vol. 202, no. 2, pp. 550-561, 2008.

[5] A. A. Kilbas, H. M. Srivastava, and J. J. Trujillo, Theory and Applications of Fractional Differential Equations, vol. 204 of North-Holland Mathematics Studies, Elsevier, Amsterdam, The Netherlands, 2006.

[6] I. Podlubny, Fractional Differential Equations, vol. 198 of Mathematics in Science and Engineering, Academic Press, San Diego, Calif, USA, 1999. 
[7] I. Podlubny, "Geometric and physical interpretation of fractional integration and fractional differentiation," Fractional Calculus \& Applied Analysis, vol. 5, no. 4, pp. 367-386, 2002.

[8] S. Zhang, "Positive solution for boundary value problem of nonlinear frctional differential equations," Electronic Journal of Differential Equations, vol. 2006, no. 36, pp. 1-12, 2006.

[9] Y. Chen and H.-L. An, "Numerical solution of coupled Burgers equations with time and space fractional derivatives," Applied Mathematics and Computation, vol. 200, no. 1, pp. 87-95, 2008.

[10] V. Gafiychuk, B. Datsko, V. Meleshko, and D. Blackmore, "Analysis of the solutions of coupled nonlinear fractional reaction-diffusion equations," Chaos, Solitons and Fractals, vol. 41, no. 3, pp. 10951104, 2009.

[11] X. Su, "Boundary value problem for a coupled system of nonlinear fractional differential equations," Applied Mathematics Letters, vol. 22, no. 1, pp. 64-69, 2009.

[12] C. Z. Bai and J. X. Fang, "The existence of a positive solution for a singular coupled system of nonlinear fractional differential equations," Applied Mathematics and Computation, vol. 150, no. 3, pp. 611-621, 2004.

[13] B. Ahmad and J. J. Nieto, "Existence results for a coupled system of nonlinear fractional differential equations with three-point boundary conditions," Computers $\mathcal{E}$ Mathematics with Applications, vol. 58, no. 9, pp. 1838-1843, 2009. 


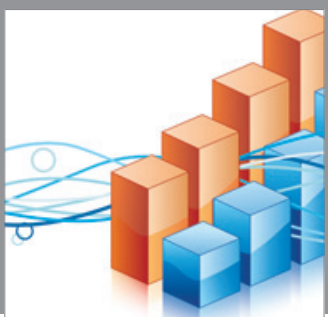

Advances in

Operations Research

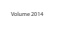

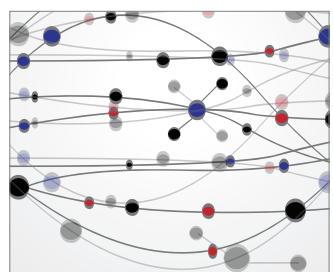

\section{The Scientific} World Journal
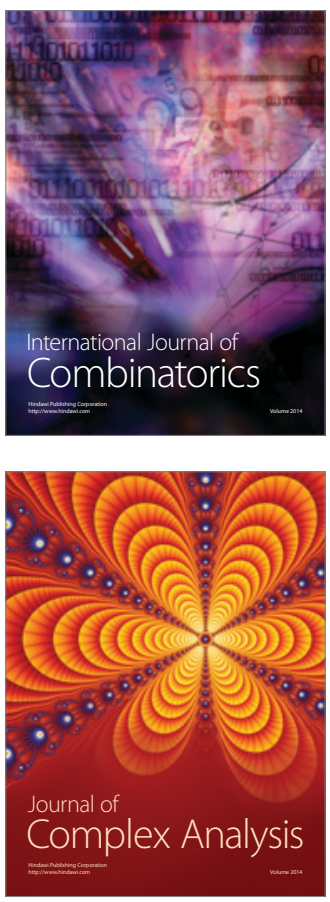

International Journal of

Mathematics and

Mathematical

Sciences
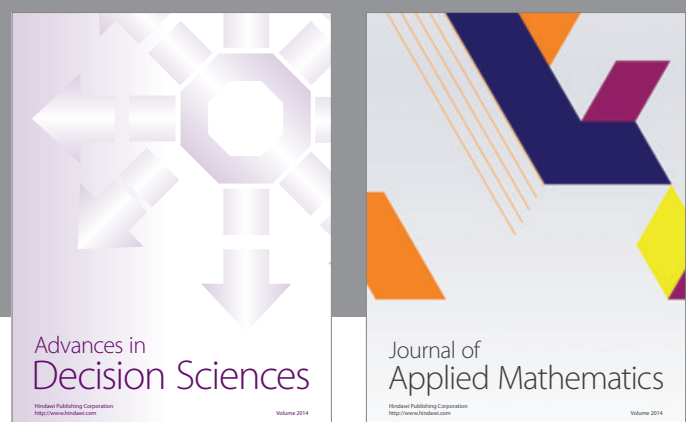

Journal of

Applied Mathematics
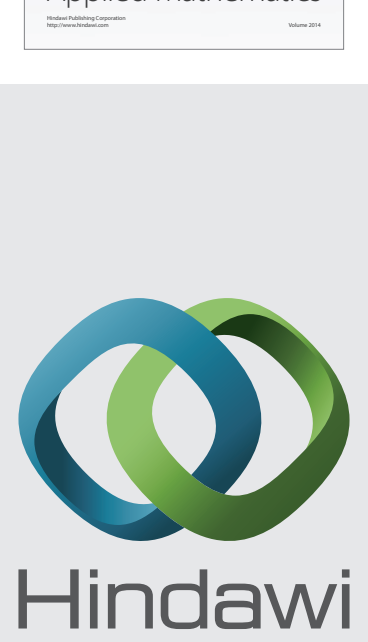

Submit your manuscripts at http://www.hindawi.com
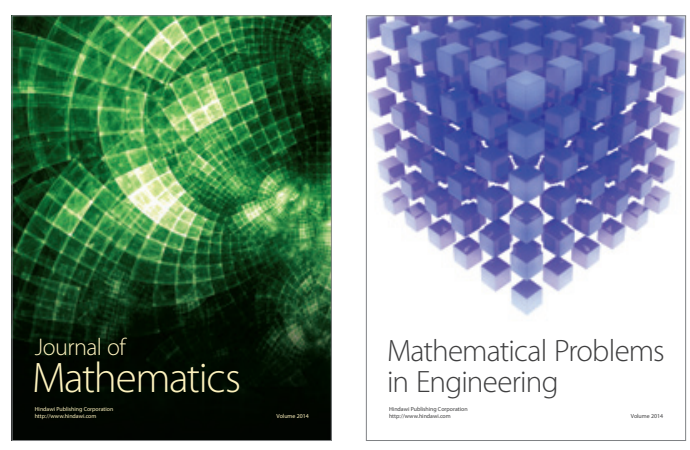

Mathematical Problems in Engineering
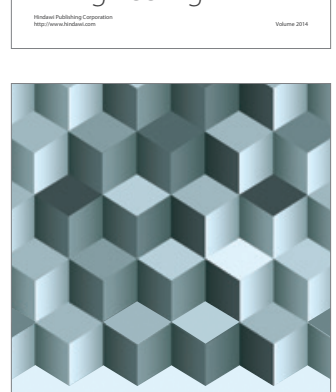

Journal of

Function Spaces
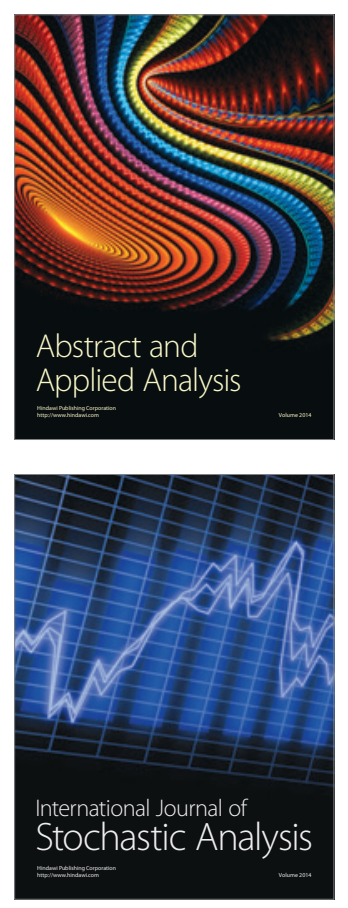

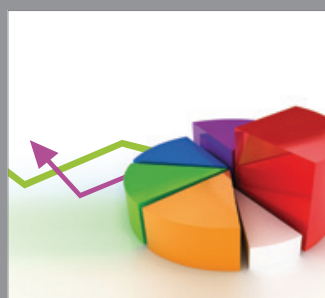

ournal of

Probability and Statistics

Promensencen
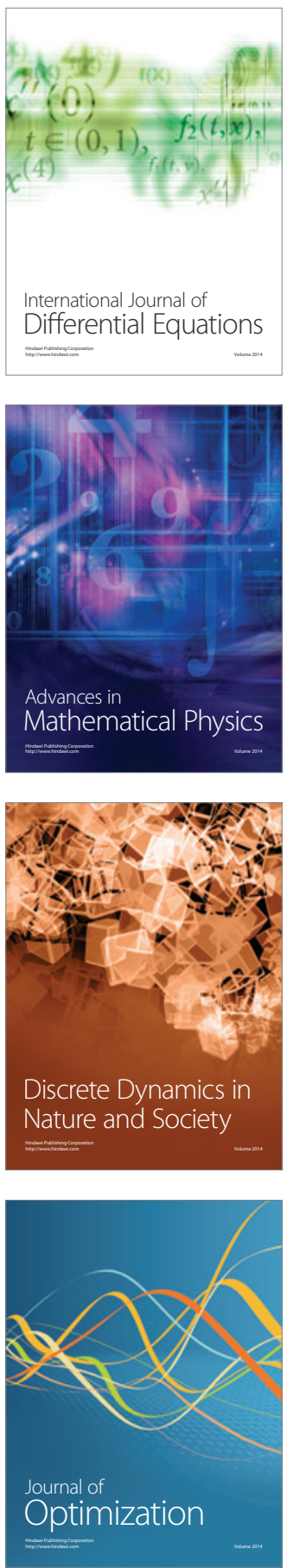